\title{
A DUALITY RELATIONSHIP FOR REGULAR CONDITIONAL RELATIVE ENTROPY
}

\author{
Li Xie, Valery A. Ugrinovskii, and Ian R. Petersen ${ }^{1}$
}

\author{
School of Information Technology and Electrical \\ Engineering, University College, The University of New \\ South Wales, Canberra, Australia. Emails: \\ \{valu,irp@ee.adfa.edu.au\}
}

\begin{abstract}
In this paper, we present a duality relationship between regular conditional free energy and regular conditional relative entropy given a sub- $\sigma$-algebra. This is achieved by using a relation between the Radon-Nikodym derivative of probability measures and that of regular conditional probability measures. Some properties of the regular conditional relative entropy under consideration are also given. The duality relation can be applied in a finite horizon robust state estimation problem for finite-alphabet hidden Markov models. Copyright ${ }^{\circledR} 2005$ IFAC
\end{abstract}

Keywords: Duality relationship, Radon-Nikodym derivative, regular conditional probability measure, regular conditional relative entropy, sub-sigma-algebra.

\section{INTRODUCTION}

The duality relationship between free energy and relative entropy such as presented in (Dupuis and Ellis, 1997) has a number of important applications in solving control problems. For example, in (Dai Pra et al., 1996), this duality was used to establish a connection between stochastic control and dynamic games, and in (Boel et al., 2002), it was used to obtain an error bound for a related error cost function defined with respect to a true probability measure. Recently, Petersen et al. (Petersen et al., 2000) and Ugrinovskii and Petersen (Ugrinovskii and Petersen, 1999) have employed the duality relationship to develop a robust control system design methodology for stochastic uncertain systems. The duality relationship between free energy and relative entropy

1 This work was supported by the Australian Research Council. also plays an important role in other research problems; e.g., see (Mitter and Newton, 2003) for an application to nonlinear estimation and (Charalambous et al., 2003) for an application to optimal disturbance attenuation for nonlinear stochastic uncertain systems.

Over recent decades, hidden Markov models (HMMs) have been studied in several research areas such as control theory (Elliott et al., 1994) and information theory (Ephraim and Merhav, 2002). It is well-known that the state estimation problem is a fundamental problem arising in relation to HMMs. In this problem, given a realized sequence of the observation process, one endeavors to estimate the unobserved sequence of the state process. In particular, one usually seeks an estimator which is optimal in some meaningful sense.

Since HMMs are essentially probabilistic models, it is desirable to consider a robust state es- 
timation problem for uncertain HMMs using a similar method to that described in (Petersen et al., 2000; Ugrinovskii and Petersen, 1999). Then, instead of the expected value of a cost functional, the conditional expectation of the error cost, given the $\sigma$-algebra generated by the observation sequence, will be used as the cost functional for the state estimation problem; see (Dey and Moore, 1995). Furthermore, the regular conditional relative entropy between regular conditional probability measures considered as an $a$ posteriori probability distance will be used as a measure of the uncertainty in uncertain HMMs.

In the HMM robust state estimation problem considered in (Xie et al., 2004), the following problem arises: Solve an unconstrained optimization problem involving maximization with respect to a class of regular conditional probability measures. This optimization problem involves conditional expectations and regular conditional relative entropy given a $\sigma$-algebra generated by the observation sequence. This motivates us to investigate the possibility as to whether the duality relationship between free energy and relative entropy can be extended to regular conditional relative entropy. This question is of interest since the duality relationship for relative entropy has been used to solve a similar optimization problem in (Petersen et al., 2000; Ugrinovskii and Petersen, 1999). Such a duality relationship may also be useful in the other control and estimation problems similar to those considered in (Boel et al., 2002; Charalambous et al., 2003; Dai Pra et al., 1996; Mitter and Newton, 2003). In this paper, we establish a duality relationship between regular conditional free energy and regular conditional relative entropy given a sub- $\sigma$-algebra by using a relation between the Radon-Nikodym derivative of probability measures and that of regular conditional probability measures. All proofs have been omitted in this conference version of the paper.

\section{PRELIMINARY RESULTS}

Let $(\Omega, \mathcal{F})$ be a measurable space. Suppose two probability measures $\mu$ and $\nu$ are defined on the same measurable space $(\Omega, \mathcal{F})$. We say a probability measure $\mu$ is absolutely continuous with respect to a probability measure $\nu$ (denoted by $\mu \ll \nu)$ if $\mu(D)=0$ whenever $D \in \mathcal{F}$ and $\nu(D)=0$. If $\mu \ll \nu$ and $\nu \ll \mu$, then $\mu$ and $\nu$ are called equivalent and this relation is indicated by $\mu \sim \nu$.

Definition 1. (Shiryayev, 1984) Let $(\Omega, \mathcal{F}, \nu)$ be a probability space and $\mathcal{G}$ be a sub- $\sigma$-algebra of $\mathcal{F}$. A regular conditional probability measure $\nu(\cdot \mid \mathcal{G})(\cdot)$ on $(\Omega, \mathcal{F})$ given $\mathcal{G}$ is a function $\nu(D \mid \mathcal{G})(\omega)$ defined for $D \in \mathcal{F}$ and $\omega \in \Omega$ such that (a) For each $\omega \in \Omega, \nu(\cdot \mid \mathcal{G})(\omega)$ is a probability measure on $\mathcal{F}$.

(b) For each $D \in \mathcal{F}, \nu(D \mid \mathcal{G})(\cdot)$ is a $\mathcal{G}$-measurable function on $\Omega$ and a version of the conditional probability of $D$ given $\mathcal{G}$. Also

$$
\int_{B} \nu(D \mid \mathcal{G})(\omega) \nu_{\mathcal{G}}(\mathrm{d} \omega)=\nu(D B), \quad \forall B \in \mathcal{G}
$$

where $\nu_{\mathcal{G}}$ is the restriction of $\nu$ to $\mathcal{G}$.

The second statement of the first sentence in Part (b) of Definition 1 implies that there exists a $\nu_{\mathcal{G}^{-}}$ null set $N$ such that for all $D \in \mathcal{F}, \nu(D \mid \mathcal{G})(\omega)=$ $\nu(D \mid \mathcal{G}), \forall \omega \in N^{c}=\Omega-N$. Here, $\nu(D \mid \mathcal{G})$ denotes the conditional probability given $\mathcal{G}$ and is a function of $\omega$. Note that the $\nu_{\mathcal{G}}$-null set $N$ is independent of the sets $D \in \mathcal{F}$.

In general, a regular conditional probability measure may not exist. However if $\mathcal{G}$ is generated by a countable partition of the sample space, then there always exists a regular conditional probability measure given $\mathcal{G}$. Also, if the sample space $\Omega$ is a Polish space (i.e., a complete separable metric space) and $\mathcal{F}$ is its Borel $\sigma$-algebra, then for any probability measure $\nu$ on $(\Omega, \mathcal{F})$ and any sub- $\sigma$ algebra $\mathcal{G} \subseteq \mathcal{F}$, a regular conditional probability measure of $\nu$ given $\mathcal{G}$ always exists; see Theorem 1.1.6 in (Stroock and Varadhan, 1979). We next suppose that the sample space $\Omega$ is a Polish space and $\mathcal{F}$ is its Borel $\sigma$-algebra. Hence $\mathcal{F}$ is separable. That is, there exists a sequence $F_{1}, F_{2}, \ldots$ of elements of $\mathcal{F}$ such that $\mathcal{F}=\sigma\left\{F_{1}, F_{2}, \ldots\right\}$.

We next summarize the relationship between the absolute continuity of probability measures and the absolute continuity of the corresponding regular conditional probability measures; see Part (d) of Proposition 1.2 in (Wu, 1997) and Lemma 4.4.7 in (Deuschel and Stroock, 1989).

Lemma 2. (a) Suppose $\mu_{\mathcal{G}} \ll \nu_{\mathcal{G}}$. If $\mu(\cdot \mid \mathcal{G})(\omega) \ll$ $\nu(\cdot \mid \mathcal{G})(\omega), \mu_{\mathcal{G}}$-a.s., then $\mu \ll \nu$.

(b) Conversely, if $\mu \ll \nu$, then $\mu(\cdot \mid \mathcal{G})(\omega) \ll$ $\nu(\cdot \mid \mathcal{G})(\omega), \mu_{\mathcal{G}}$-a.s.

Suppose $\mu \ll \nu$. It follows from Part (b) of Lemma 2 that there exists a $\mu_{\mathcal{G}}$-null set denoted by $\Lambda \in \mathcal{G}$ such that for each $\omega \in \Lambda^{c}, \mu(\cdot \mid \mathcal{G})(\omega) \ll \nu(\cdot \mid \mathcal{G})(\omega)$. We now define a family of probability measures $\check{\mu}(\cdot, \omega)$ parameterized by $\omega \in \Omega$ such that

$$
\check{\mu}(\cdot, \omega)=\left\{\begin{array}{l}
\mu(\cdot \mid \mathcal{G})(\omega), \text { if } \omega \in \Lambda^{c} ; \\
\nu(\cdot \mid \mathcal{G})(\omega), \text { otherwise. }
\end{array}\right.
$$

Hence for each $\omega, \check{\mu}(\cdot, \omega) \ll \nu(\cdot \mid \mathcal{G})(\omega)$. Also for each $D \in \mathcal{F}, \check{\mu}(D, \omega)$ is $\mathcal{G}$-measurable. Under the condition that the $\sigma$-algebra $\mathcal{F}$ is separable, using the Martingale Convergence Theorem, we can construct a nonnegative $\mathcal{F} \times \mathcal{G}$-measurable function $f\left(\omega^{\prime}, \omega\right)$ such that for each $\omega$, 


$$
\check{\mu}(D, \omega)=\int_{D} f\left(\omega^{\prime}, \omega\right) \nu\left(\mathrm{d} \omega^{\prime} \mid \mathcal{G}\right)(\omega) ;
$$

see Theorem V.58 in (Dellacherie and Meyer, 1982). Here $\mathcal{F} \times \mathcal{G}=\sigma\left\{A_{1} \times A_{2}: A_{1} \in \mathcal{F}, A_{2} \in \mathcal{G}\right\}$. Then it follows from (1) and (2) that for each $\omega \in \Lambda^{c}, \forall D \in \mathcal{F}$,

$$
\mu(D \mid \mathcal{G})(\omega)=\int_{D} \frac{\mathrm{d} \mu(\cdot \mid \mathcal{G})}{\mathrm{d} \nu(\cdot \mid \mathcal{G})}\left(\omega^{\prime}, \omega\right) \nu\left(\mathrm{d} \omega^{\prime} \mid \mathcal{G}\right)(\omega)
$$

Here, $\frac{\mathrm{d} \mu(\cdot \mid \mathcal{G})}{\mathrm{d} \nu(\cdot \mid \mathcal{G})}\left(\omega^{\prime}, \omega\right) \triangleq f\left(\omega^{\prime}, \omega\right)$ and is called the Radon-Nikodym derivative of the regular conditional probability measure $\mu(\cdot \mid \mathcal{G})(\omega)$ with respect to $\nu(\cdot \mid \mathcal{G})(\omega)$. Furthermore, $(3)$ implies that for all $D \in \mathcal{F}$,

$$
\begin{aligned}
& \mu(D \mid \mathcal{G})(\omega) \\
& =\int_{D} \frac{\mathrm{d} \mu(\cdot \mid \mathcal{G})}{\mathrm{d} \nu(\cdot \mid \mathcal{G})}\left(\omega^{\prime}, \omega\right) \nu\left(\mathrm{d} \omega^{\prime} \mid \mathcal{G}\right)(\omega), \quad \mu_{\mathcal{G}} \text {-a.s. }
\end{aligned}
$$

We next present a chain rule for the regular conditional probability measure.

Lemma 3. Suppose $\mu \ll \mu_{0} \ll \nu$ as probability measures defined on $(\Omega, \mathcal{F})$.

(a) If $\bar{g}\left(\omega^{\prime}, \omega\right)$ is an $\mathcal{F} \times \mathcal{G}$-measurable function from $\Omega \times \Omega$ to the extended real line whose integral $\int_{\Omega} g\left(\omega^{\prime}, \omega\right) \mu_{0}\left(\mathrm{~d} \omega^{\prime} \mid \mathcal{G}\right)(\omega)$ exists for any $\omega \in \Omega$, then for any $D \in \mathcal{F}$,

$$
\begin{aligned}
& \int_{D} \bar{g}\left(\omega^{\prime}, \omega\right) \mu_{0}\left(\mathrm{~d} \omega^{\prime} \mid \mathcal{G}\right)(\omega)= \\
& \int_{D} \bar{g}\left(\omega^{\prime}, \omega\right) \frac{\mathrm{d} \mu_{0}(\cdot \mid \mathcal{G})}{\mathrm{d} \nu(\cdot \mid \mathcal{G})}\left(\omega^{\prime}, \omega\right) \nu\left(\mathrm{d} \omega^{\prime} \mid \mathcal{G}\right)(\omega), \mu_{0 \mathcal{G}} \text {-a.s. } \\
& \text { (b) } \frac{\mathrm{d} \mu(\cdot \mid \mathcal{G})}{\mathrm{d} \nu(\cdot \mid \mathcal{G})}\left(\omega^{\prime}, \omega\right) \\
& =\frac{\mathrm{d} \mu(\cdot \mid \mathcal{G})}{\mathrm{d} \mu_{0}(\cdot \mid \mathcal{G})}\left(\omega^{\prime}, \omega\right) \frac{\mathrm{d} \mu_{0}(\cdot \mid \mathcal{G})}{\mathrm{d} \nu(\cdot \mid \mathcal{G})}\left(\omega^{\prime}, \omega\right), \\
& \nu(\cdot \mid \mathcal{G})(\omega) \text {-a.s. and } \mu_{\mathcal{G}} \text { - } \omega \text {-a.s. }
\end{aligned}
$$

Here, the last equality holds for all $\omega \in \Theta \in \mathcal{G}$ and $\omega^{\prime} \in \Pi(\omega) \in \mathcal{F}$, where the set $\Theta$ satisfies $\mu_{\mathcal{G}}(\Theta)=1$ and $\nu(\Pi(\omega) \mid \mathcal{G})(\omega)=1, \forall \omega \in \Theta$.

We use the Radon-Nikodym derivative given by (2) to define the regular conditional relative entropy between regular conditional probability measures.

Definition 4. Consider two probability measures $\mu$ and $\nu$ on $(\Omega, \mathcal{F})$ satisfying $\mu \ll \nu$. Let $\Lambda$ denote the $\mu_{\mathcal{G}}$-null set such that $\forall \omega \in \Lambda^{c}, \mu(\cdot \mid \mathcal{G})(\omega) \ll$ $\nu(\cdot \mid \mathcal{G})(\omega)$. Then the regular conditional relative entropy of $\mu(\cdot \mid \mathcal{G})(\omega)$ with respect to $\nu(\cdot \mid \mathcal{G})(\omega)$ is defined by

$$
\begin{aligned}
& \mathcal{R}(\mu(\cdot \mid \mathcal{G}) \| \nu(\cdot \mid \mathcal{G}))(\omega) \\
& \triangleq \begin{cases}\mathbf{E}^{\mu(\cdot \mid \mathcal{G})(\omega)}\left[\log \frac{\mathrm{d} \mu(\cdot \mid \mathcal{G})}{\mathrm{d} \nu(\cdot \mid \mathcal{G})}\left(\omega^{\prime}, \omega\right)\right], & \text { if } \omega \in \Lambda^{c} ; \\
+\infty, & \text { otherwise }\end{cases}
\end{aligned}
$$

where $\frac{\mathrm{d} \mu(\cdot \mid \mathcal{G})}{\mathrm{d} \nu(\cdot \mid \mathcal{G})}\left(\omega^{\prime}, \omega\right)$ is the Radon-Nikodym derivative of $\mu(\cdot \mid \mathcal{G})(\omega)$ with respect to $\nu(\cdot \mid \mathcal{G})(\omega)$.

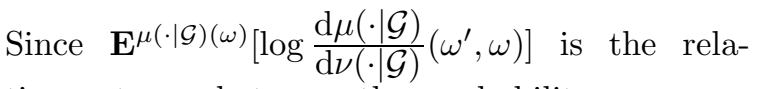
tive entropy between the probability measures $\mu(\cdot \mid \mathcal{G})(\omega)$ and $\nu(\cdot \mid \mathcal{G})(\omega)$ for a fixed $\omega \in \Lambda^{c}$, it exists on the extended real line; see Section 1.4 in (Dupuis and Ellis, 1997). This implies that $\mathcal{R}(\mu(\cdot \mid \mathcal{G}) \| \nu(\cdot \mid \mathcal{G}))(\omega)$ is well-defined. Furthermore, $\mathbf{E}^{\mu(\cdot \mid \mathcal{G})(\omega)}\left[\log \frac{\mathrm{d} \mu(\cdot \mid \mathcal{G})}{\mathrm{d} \nu(\cdot \mid \mathcal{G})}\left(\omega^{\prime}, \omega\right)\right]$ as a function of $\omega$ is $\mathcal{G}$-measurable. This follows from the fact that the regular conditional probability measure $\mu(\cdot \mid \mathcal{G})(\omega)$ is a stochastic (or probability) kernel from $(\Omega, \mathcal{G})$ to $(\Omega, \mathcal{F})$ and $\log \frac{\mathrm{d} \mu(\cdot \mid \mathcal{G})}{\mathrm{d} \nu(\cdot \mid \mathcal{G})}\left(\omega^{\prime}, \omega\right)$ is $\mathcal{F} \times \mathcal{G}$-measurable; see Lemma 1.38 in (Kallenberg, 1997). Hence $\mathcal{R}(\mu(\cdot \mid \mathcal{G}) \| \nu(\cdot \mid \mathcal{G}))(\omega)$ is a $\mathcal{G}$-measurable function from $\Omega$ into the extended real line since for each $\omega \in \Lambda$ it is defined to be $+\infty$.

We will use the following equality about the Radon-Nikodym derivative between regular conditional probability measures to derive our results:

$$
\begin{aligned}
\frac{\mathrm{d} \mu}{\mathrm{d} \nu}\left(\omega^{\prime}\right)= & \frac{\mathrm{d} \mu_{\mathcal{G}}}{\mathrm{d} \nu_{\mathcal{G}}}(\omega) \frac{\mathrm{d} \mu(\cdot \mid \mathcal{G})}{\mathrm{d} \nu(\cdot \mid \mathcal{G})}\left(\omega^{\prime}, \omega\right), \\
& \nu(\cdot \mid \mathcal{G})(\omega) \text {-a.s. and } \mu_{\mathcal{G}}-\omega \text {-a.s. }
\end{aligned}
$$

where $\mu \ll \nu$. The proof of equality (4) is included in the full version of this paper. Other related equalities concerning the Radon-Nikodym derivative between regular conditional probability measures can be found in Lemma 4.4.7 in (Deuschel and Stroock, 1989). Then, $\mathcal{R}(\mu(\cdot \mid \mathcal{G}) \| \nu(\cdot \mid \mathcal{G}))(\omega)$ can be written as

$$
\begin{aligned}
& \mathcal{R}(\mu(\cdot \mid \mathcal{G}) \| \nu(\cdot \mid \mathcal{G}))(\omega) \\
& =\mathbf{E}^{\mu}\left[\log \frac{\mathrm{d} \mu}{\mathrm{d} \nu} \mid \mathcal{G}\right](\omega)-\log \mathbf{E}^{\nu}\left[\frac{\mathrm{d} \mu}{\mathrm{d} \nu} \mid \mathcal{G}\right](\omega), \quad \mu_{\mathcal{G}} \text {-a.s. }
\end{aligned}
$$

The next lemma will give some properties of regular conditional relative entropy.

Lemma 5. (a) $\forall \omega \in \Omega, \mathcal{R}(\mu(\cdot \mid \mathcal{G}) \| \nu(\cdot \mid \mathcal{G}))(\omega) \geq 0$.

(b) $\mathcal{R}(\mu(\cdot \mid \mathcal{G}) \| \nu(\cdot \mid \mathcal{G}))(\omega)=0, \mu_{\mathcal{G}}$-a.s. if and only if $\mu(\cdot \mid \mathcal{G})(\omega)=\nu(\cdot \mid \mathcal{G})(\omega), \mu_{\mathcal{G}}$-a.s.

Let $\mathcal{P}(\Omega, \mathcal{F}, \mathcal{G}, \nu)$ denote the set of all probability measures on $(\Omega, \mathcal{F})$ which are absolutely continuous with respect to $\nu$ and whose restrictions to $\mathcal{G}$ and $\nu_{\mathcal{G}}$ are equivalent in the sense of absolute continuity. That is, if $\mu \in \mathcal{P}(\Omega, \mathcal{F}, \mathcal{G}, \nu)$, then $\mu \ll \nu$ and $\mu_{\mathcal{G}} \sim \nu_{\mathcal{G}}$. The probability measure $\nu$ is called the reference probability measure and is fixed. Let $\mathcal{P}(\Omega, \mathcal{F}, \nu \mid \mathcal{G})$ denote the set of all regular conditional probability measures defined by

$\mathcal{P}(\Omega, \mathcal{F}, \nu \mid \mathcal{G})(\omega) \triangleq\{\mu(\cdot \mid \mathcal{G})(\omega): \mu \in \mathcal{P}(\Omega, \mathcal{F}, \mathcal{G}, \nu)\}$.

Note that if $\mu(\cdot \mid \mathcal{G})(\omega) \in \mathcal{P}(\Omega, \mathcal{F}, \nu \mid \mathcal{G})(\omega)$, then $\mu_{\mathcal{G}} \sim \nu_{\mathcal{G}}$ and $\mu \ll \nu$. Hence, properties which 
hold $\mu_{\mathcal{G}}$-a.s. also hold $\nu_{\mathcal{G}}$-a.s. and vice versa.

$$
\begin{aligned}
\text { Let } & \overline{\mathcal{P}}(\Omega, \mathcal{F}, \nu \mid \mathcal{G})(\omega) \\
& \triangleq\{\mu(\cdot \mid \mathcal{G})(\omega): \mu(\cdot \mid \mathcal{G})(\omega) \in \mathcal{P}(\Omega, \mathcal{F}, \nu \mid \mathcal{G})(\omega) \\
& \text { and } \left.\mathcal{R}(\mu(\cdot \mid \mathcal{G}) \| \nu(\cdot \mid \mathcal{G}))(\omega)<\infty, \nu_{\mathcal{G}} \text {-a.s. }\right\} .
\end{aligned}
$$

We first define the (almost sure) convexity of the set $\overline{\mathcal{P}}(\Omega, \mathcal{F}, \nu \mid \mathcal{G})(\omega)$. Then the strict convexity of the regular conditional relative entropy is defined on the set $\overline{\mathcal{P}}(\Omega, \mathcal{F}, \nu \mid \mathcal{G})(\omega)$ in the sense of almost sure under $\nu_{\mathcal{G}}$.

Definition 6. We say that the set $\overline{\mathcal{P}}(\Omega, \mathcal{F}, \nu \mid \mathcal{G})(\omega)$ is convex if the following property holds:

For given $\mu_{1}(\cdot \mid \mathcal{G})(\omega), \mu_{2}(\cdot \mid \mathcal{G})(\omega)$ in $\overline{\mathcal{P}}(\Omega, \mathcal{F}, \nu \mid \mathcal{G})(\omega)$ and $\lambda \in(0,1)$, there exists a probability measure $\hat{\mu}$ on $(\Omega, \mathcal{F})$ whose regular conditional probability measure $\hat{\mu}(\cdot \mid \mathcal{G})(\omega) \in \overline{\mathcal{P}}(\Omega, \mathcal{F}, \nu \mid \mathcal{G})(\omega)$ satisfies $\hat{\mu}(\cdot \mid \mathcal{G})(\omega)=\mu_{1}(\cdot \mid \mathcal{G})(\omega)+(1-\lambda) \mu_{2}(\cdot \mid \mathcal{G})(\omega), \nu_{\mathcal{G}}$-a.s. That is, there exists a $\nu_{\mathcal{G}}$-null set $N$ such that for each $D \in \mathcal{F}$ and $\forall \omega \in N^{c}$,

$$
\hat{\mu}(D \mid \mathcal{G})(\omega)=\mu_{1}(D \mid \mathcal{G})(\omega)+(1-\lambda) \mu_{2}(D \mid \mathcal{G})(\omega) .
$$

This property is denoted by

$\lambda \mu_{1}(\cdot \mid \mathcal{G})(\omega)+(1-\lambda) \mu_{2}(\cdot \mid \mathcal{G})(\omega) \in \overline{\mathcal{P}}(\Omega, \mathcal{F}, \nu \mid \mathcal{G})(\omega)$.

Definition 7. We say that the regular conditional relative entropy $\mathcal{R}(\mu(\cdot \mid \mathcal{G}) \| \nu(\cdot \mid \mathcal{G}))(\omega)$ is a strictly convex function of $\mu(\cdot \mid \mathcal{G})(\omega)$ on the set $\overline{\mathcal{P}}(\Omega, \mathcal{F}, \nu \mid \mathcal{G})(\omega)$ in the sense of almost sure under $\nu_{\mathcal{G}}$ if the following property holds:

For given $\mu_{1}(\cdot \mid \mathcal{G})(\omega), \mu_{2}(\cdot \mid \mathcal{G})(\omega)$ in $\overline{\mathcal{P}}(\Omega, \mathcal{F}, \nu \mid \mathcal{G})(\omega)$ satisfying $\mu_{1}(\cdot \mid \mathcal{G})(\omega) \neq \mu_{2}(\cdot \mid \mathcal{G})(\omega)$, $\nu_{\mathcal{G}}$-a.s., and $\lambda \in(0,1)$,

$$
\begin{gathered}
\mathcal{R}\left(\lambda \mu_{1}(\cdot \mid \mathcal{G})(\omega)+(1-\lambda) \mu_{2}(\cdot \mid \mathcal{G})(\omega) \| \nu(\cdot \mid \mathcal{G})\right)(\omega) \\
<\lambda \mathcal{R}\left(\mu_{1}(\cdot \mid \mathcal{G}) \| \nu(\cdot \mid \mathcal{G})\right)(\omega)+(1-\lambda) \\
\mathcal{R}\left(\mu_{2}(\cdot \mid \mathcal{G}) \| \nu(\cdot \mid \mathcal{G})\right)(\omega), \quad \nu_{\mathcal{G}} \text {-a.s. }
\end{gathered}
$$

Lemma 8. With the above definitions,

(a) The set $\overline{\mathcal{P}}(\Omega, \mathcal{F}, \nu \mid \mathcal{G})(\omega)$ is convex.

(b) $\mathcal{R}(\mu(\cdot \mid \mathcal{G}) \| \nu(\cdot \mid \mathcal{G}))(\omega)$ is a strictly convex function of $\mu(\cdot \mid \mathcal{G})(\omega) \nu_{\mathcal{G}}$-a.s. on $\overline{\mathcal{P}}(\Omega, \mathcal{F}, \nu \mid \mathcal{G})(\omega)$.

\section{A DUALITY RELATIONSHIP FOR REGULAR CONDITIONAL RELATIVE ENTROPY}

In order to state our main result, we first introduce a measurable function $g(\omega)$ mapping $\Omega$ into the extended real line. Also, we assume that $g$ is either bounded from below or bounded from above. Then under this assumption, the expectation of $g$ exists, and so does the conditional expectation of $g$ given $\mathcal{G}$ with respect to any probability measure. Furthermore for each $\mu(\cdot \mid \mathcal{G})(\omega) \in \overline{\mathcal{P}}(\Omega, \mathcal{F}, \nu \mid \mathcal{G})(\omega)$, we may assume that $\mathcal{R}(\mu(\cdot \mid \mathcal{G}) \| \nu(\cdot \mid \mathcal{G}))(\omega)+\mathbf{E}^{\mu}[g \mid \mathcal{G}](\omega)$ is well-defined for each $\omega$. This follows since outside of a $\nu_{\mathcal{G}}$-null set, the regular conditional relative entropy satisfies $\mathcal{R}(\mu(\cdot \mid \mathcal{G}) \| \nu(\cdot \mid \mathcal{G}))(\omega)<$ $\infty$. Then for each $\mu(\cdot \mid \mathcal{G})(\omega) \in \overline{\mathcal{P}}(\Omega, \mathcal{F}, \nu \mid \mathcal{G})(\omega)$, $\mathcal{R}(\mu(\cdot \mid \mathcal{G}) \| \nu(\cdot \mid \mathcal{G}))(\omega)+\mathbf{E}^{\mu}[g \mid \mathcal{G}](\omega)$ is $\mathcal{G}$-measurable. We next give a definition of the notion of essential infimum. There is a similar definition for the essential supremum of a family of measurable functions in (Chow and Teicher, 1988) for a fixed probability measure.

Definition 9. The essential infimum $h(\omega)$ of the family $\left\{\mathcal{R}(\mu(\cdot \mid \mathcal{G}) \| \nu(\cdot \mid \mathcal{G}))(\omega)+\mathbf{E}^{\mu}[g \mid \mathcal{G}](\omega), \mu(\cdot \mid \mathcal{G})(\omega)\right.$ $\in \overline{\mathcal{P}}(\Omega, \mathcal{F}, \nu \mid \mathcal{G})(\omega)\}$, denoted by

$$
\begin{aligned}
h(\omega)= & \underset{\mu(\cdot \mid \mathcal{G})(\omega) \in \overline{\mathcal{P}}(\Omega, \mathcal{F}, \nu \mid \mathcal{G})(\omega)}{\operatorname{einf}} \\
& \left\{\mathcal{R}(\mu(\cdot \mid \mathcal{G}) \| \nu(\cdot \mid \mathcal{G}))(\omega)+\mathbf{E}^{\mu}[g \mid \mathcal{G}](\omega)\right\},
\end{aligned}
$$

is defined by the properties:

(a) $h(\omega)$ is $\mathcal{G}$-measurable;

(b) $\mathcal{R}(\mu(\cdot \mid \mathcal{G}) \| \nu(\cdot \mid \mathcal{G}))(\omega)+\mathbf{E}^{\mu}[g \mid \mathcal{G}](\omega) \geq h(\omega), \nu_{\mathcal{G}}$ a.s., $\forall \mu(\cdot \mid \mathcal{G})(\omega) \in \overline{\mathcal{P}}(\Omega, \mathcal{F}, \nu \mid \mathcal{G})(\omega)$;

(c) for any $\hat{h}(\omega)$ satisfying (a) and (b), $\hat{h}(\omega) \leq$ $h(\omega), \nu_{\mathcal{G}}$-a.s.

Clearly, if such an $h(\omega)$ exists, then it is unique in the sense that two such essential infima are equal $\nu_{\mathcal{G}}$-a.s..

Lemma 10. With the above definitions, there exists a countable subset $\overline{\mathcal{P}}_{0}(\Omega, \mathcal{F}, \nu \mid \mathcal{G})(\omega)$ of $\overline{\mathcal{P}}(\Omega, \mathcal{F}, \nu \mid \mathcal{G})(\omega)$ such that

$$
\begin{aligned}
\inf _{\mu(\cdot \mid \mathcal{G})(\omega) \in \overline{\mathcal{P}}_{0}(\Omega, \mathcal{F}, \nu \mid \mathcal{G})(\omega)}\{\mathcal{R}(\mu(\cdot \mid \mathcal{G}) \| \nu(\cdot \mid \mathcal{G}))(\omega) \\
\left.+\mathbf{E}^{\mu}[g \mid \mathcal{G}](\omega)\right\}=h(\omega) .
\end{aligned}
$$

Let $\mathbb{N}$ denote the set of all positive integers. Also let $a \wedge b=\min (a, b)$ and $a \vee b=\max (a, b)$. We next give a definition of the notion of lattice property.

Definition 11. A set $\left\{\psi_{\gamma}, \gamma \in \Gamma\right\}$ is said to have the lattice property if given $\gamma_{1}, \gamma_{2} \in \Gamma$, then there exist $\gamma_{3}, \gamma_{4} \in \Gamma$ such that

$$
\psi_{\gamma_{3}}=\psi_{\gamma_{1}} \wedge \psi_{\gamma_{2}}, \quad \psi_{\gamma_{4}}=\psi_{\gamma_{1}} \vee \psi_{\gamma_{2}} \text {, a.s. }
$$

Lemma 12. The set

$$
\begin{array}{r}
\left\{\mathcal{R}(\mu(\cdot \mid \mathcal{G}) \| \nu(\cdot \mid \mathcal{G}))(\omega)+\mathbf{E}^{\mu}[g \mid \mathcal{G}](\omega)\right. \\
\mu(\cdot \mid \mathcal{G})(\omega) \in \overline{\mathcal{P}}(\Omega, \mathcal{F}, \nu \mid \mathcal{G})(\omega)\}
\end{array}
$$

has the lattice property. Also, there exists a decreasing sequence

$$
\begin{array}{r}
\left\{\mathcal{R}\left(\mu_{n}(\cdot \mid \mathcal{G}) \| \nu(\cdot \mid \mathcal{G})\right)(\omega)+\mathbf{E}^{\mu_{n}}[g \mid \mathcal{G}](\omega)\right. \\
\left.\mu_{n}(\cdot \mid \mathcal{G})(\omega) \in \overline{\mathcal{P}}(\Omega, \mathcal{F}, \nu \mid \mathcal{G})(\omega)\right\}
\end{array}
$$

such that 


$$
\begin{aligned}
& \lim _{n \rightarrow \infty} \mathcal{R}\left(\mu_{n}(\cdot \mid \mathcal{G}) \| \nu(\cdot \mid \mathcal{G})\right)(\omega)+\mathbf{E}^{\mu_{n}}[g \mid \mathcal{G}](\omega)= \\
& \underset{\mu(\cdot \mid \mathcal{G})(\omega) \in \overline{\mathcal{P}}(\Omega, \mathcal{F}, \nu \mid \mathcal{G})(\omega)}{\operatorname{einf}}\{\mathcal{R}(\mu(\cdot \mid \mathcal{G}) \| \nu(\cdot \mid \mathcal{G}))(\omega) \\
& \left.+\mathbf{E}^{\mu}[g \mid \mathcal{G}](\omega)\right\}, \quad \nu_{\mathcal{G}} \text {-a.s. }
\end{aligned}
$$

If $g(\omega)$ is a bounded measurable function, then the essential infimum (6) can be attained in the set $\overline{\mathcal{P}}(\Omega, \mathcal{F}, \nu \mid \mathcal{G})(\omega)$.

Theorem 13. Let $g(\omega)$ be a bounded measurable function. Then the following conclusions hold:

(a) We have the variational formula

$$
\begin{aligned}
& -\log \mathbf{E}^{\nu}\left[e^{-g} \mid \mathcal{G}\right](\omega) \\
& =\operatorname{einf}_{\mu(\cdot \mid \mathcal{G})(\omega) \in \overline{\mathcal{P}}(\Omega, \mathcal{F}, \nu \mid \mathcal{G})(\omega)}\{\mathcal{R}(\mu(\cdot \mid \mathcal{G}) \| \nu(\cdot \mid \mathcal{G}))(\omega) \\
& \left.\quad+\mathbf{E}^{\mu}[g \mid \mathcal{G}](\omega)\right\} .
\end{aligned}
$$

(b) Let $\mu_{0}$ be a probability measure on $(\Omega, \mathcal{F})$ satisfying

$$
\frac{\mathrm{d} \mu_{0}}{\mathrm{~d} \nu}(\omega)=e^{-g(\omega)} \frac{1}{\mathbf{E}^{\nu}\left[e^{-g(\omega)}\right]}, \quad \forall \omega \in \Omega .
$$

Then the infimum in the variational formula (7) is attained uniquely at $\mu_{0}(\cdot \mid \mathcal{G})(\omega)$.

\section{A SPECIAL CASE}

In this section, we consider a special case in which the sub- $\sigma$-algebra $\mathcal{G}$ is generated by a countable partition of the sample space $\Omega$. Let $\mathcal{G}$ be a sub$\sigma$-algebra of $\mathcal{F}$ satisfying

$$
\mathcal{G}=\sigma\left\{B_{1}, B_{2}, \ldots\right\}
$$

where $\Omega=\cup_{i} B_{i}$, each $B_{i}$ is non-empty set, and $B_{i} \cap B_{j}=\emptyset$ for $i \neq j$. Such $B_{1}, B_{2}, \ldots$ define a countable partition of $\Omega$ and each $B_{i}$ is called an atom of $\mathcal{G}$. Such a $\mathcal{G}$ can be considered to be generated by a sequence of random variables with finite or countable range; e.g., in a finite horizon robust state estimation problem for finitealphabet hidden Markov models, such a $\mathcal{G}$ is generated by the observation sequence. It is obvious that $\mathcal{G}$ is separable.

Consider the reference probability measure $\nu$ on $(\Omega, \mathcal{F})$. For each $D \in \mathcal{F}$, we define a function for $\omega \in \Omega$ and $D \in \mathcal{F}$ as follows:

$$
\nu(D \mid \mathcal{G})(\omega)= \begin{cases}\frac{\nu\left(D B_{i}\right)}{\nu\left(B_{i}\right)}, & \text { if } \omega \in B_{i}, \nu\left(B_{i}\right)>0 \\ \nu(D), & \text { if } \omega \in B_{i}, \nu\left(B_{i}\right)=0 .\end{cases}
$$

Hence $\nu(\cdot \mid \mathcal{G})(\cdot)$ is a regular conditional probability measure relative to $\mathcal{F}$ given $\mathcal{G}$. Next the regular conditional probability measure of any probability measure on $(\Omega, \mathcal{F})$ given $\mathcal{G}$ is defined by $(9)$.

Let $\mathcal{A}$ denote the class of all atoms with positive probability under $\nu_{\mathcal{G}}$. Also, let $\overline{\mathcal{A}}$ denote the class of all atoms with zero probability under $\nu_{\mathcal{G}}$. Let $\nu\left(\cdot \mid B_{i}\right)$ denote the conditional probability measure with respect to $\nu$ given $B_{i} \in \mathcal{A}$. It follows from (9) that the regular conditional probability measure $\nu(D \mid \mathcal{G})(\omega)$ for each $D \in \mathcal{F}$ and $\omega \in \Omega$ can be written as

$$
\begin{aligned}
& \nu(D \mid \mathcal{G})(\omega) \\
& =\sum_{B_{i} \in \mathcal{A}} \nu\left(D \mid B_{i}\right) I_{B_{i}}(\omega)+\sum_{B_{i} \in \overline{\mathcal{A}}} \nu(D) I_{B_{i}}(\omega) .
\end{aligned}
$$

Also for any $\omega \in B_{i} \in \mathcal{A}, \nu(\cdot \mid \mathcal{G})(\omega)=\nu\left(\cdot \mid B_{i}\right)$.

Let $\mu$ be another probability measure defined on $(\Omega, \mathcal{F})$ satisfying $\mu \ll \nu$. Let $\mathcal{A}_{\mu_{\mathcal{G}}}$ denote the class of all atoms with positive probability under $\mu_{\mathcal{G}}$. Also, let $\mathcal{A}_{\mu_{\mathcal{G}}}^{0}$ be the union of all atoms with zero probability under $\mu_{\mathcal{G}}$. It is obvious that $\mu_{\mathcal{G}}\left(\mathcal{A}_{\mu_{\mathcal{G}}}^{0}\right)=0$ since the number of the atoms of $\mathcal{G}$ is countable.

Without the assumption that $\mathcal{F}$ is separable, the next lemma will state the relationship between the absolute continuity of probability measures and the absolute continuity of the corresponding regular conditional probability measures for the case in which the sub- $\sigma$-algebra $\mathcal{G}$ is generated by a countable partition of the sample space.

Lemma 14. $\mu(\cdot \mid \mathcal{G})(\omega) \ll \nu(\cdot \mid \mathcal{G})(\omega), \mu_{\mathcal{G}}$-a.s. if and only if $\mu \ll \nu$.

Based on the sufficiency part of Lemma 14, we now define the Radon-Nikodym derivative of $\mu(\cdot \mid \mathcal{G})(\omega)$ with respect to $\nu(\cdot \mid \mathcal{G})(\omega)$ by

$$
\begin{aligned}
& \frac{\mathrm{d} \mu(\cdot \mid \mathcal{G})}{\mathrm{d} \nu(\cdot \mid \mathcal{G})}\left(\omega^{\prime}, \omega\right) \\
& = \begin{cases}\frac{\mathrm{d} \mu\left(\cdot \mid B_{i}\right)}{\mathrm{d} \nu\left(\cdot \mid B_{i}\right)}\left(\omega^{\prime}\right), & \forall \omega \in B_{i} \in \mathcal{A}_{\mu_{\mathcal{G}}} \text { and } \omega^{\prime} \in \Omega ; \\
c, & \forall \omega \in \mathcal{A}_{\mu_{\mathcal{G}}}^{0} \text { and } \omega^{\prime} \in \Omega .\end{cases}
\end{aligned}
$$

Here $\frac{\mathrm{d} \mu\left(\cdot \mid B_{i}\right)}{\mathrm{d} \nu\left(\cdot \mid B_{i}\right)}$ is the Radon-Nikodym derivative of $\mu\left(\cdot \mid B_{i}\right)$ with respect to $\nu\left(\cdot \mid B_{i}\right)$ and $c$ is a nonnegative constant. It is obvious that $\frac{\mathrm{d} \mu(\cdot \mid \mathcal{G})}{\mathrm{d} \nu(\cdot \mid \mathcal{G})}\left(\omega^{\prime}, \omega\right)$ is $\mathcal{F} \times \mathcal{G}$-measurable. Then

$\mu(D \mid \mathcal{G})(\omega)=\int_{D} \frac{\mathrm{d} \mu(\cdot \mid \mathcal{G})}{\mathrm{d} \nu(\cdot \mid \mathcal{G})}\left(\omega^{\prime}, \omega\right) \nu\left(\mathrm{d} \omega^{\prime} \mid \mathcal{G}\right)(\omega), \mu_{\mathcal{G}}$-a.s.

Furthermore, the regular conditional relative entropy of $\mu(\cdot \mid \mathcal{G})(\omega)$ with respect to $\nu(\cdot \mid \mathcal{G})(\omega)$, $\mathcal{R}(\mu(\cdot \mid \mathcal{G}) \| \nu(\cdot \mid \mathcal{G}))(\omega)$ given $\mathcal{G}$, is defined by

$\mathcal{R}(\mu(\cdot \mid \mathcal{G}) \| \nu(\cdot \mid \mathcal{G}))(\omega)$

$\triangleq \begin{cases}\mathbf{E}^{\mu(\cdot \mid \mathcal{G})(\omega)}\left[\log \frac{\mathrm{d} \mu(\cdot \mid \mathcal{G})}{\mathrm{d} \nu(\cdot \mid \mathcal{G})}\left(\omega^{\prime}, \omega\right)\right], & \text { if } \omega \in \Omega-\mathcal{A}_{\mu_{\mathcal{G}}}^{0} ; \\ +\infty, & \text { otherwise }\end{cases}$

As before let $\mathcal{P}(\Omega, \mathcal{F}, \mathcal{G}, \nu)$ denote the set of all probability measures on $(\Omega, \mathcal{F})$ which are absolutely continuous with respect to $\nu$ and whose 
restrictions to $\mathcal{G}$ and $\nu_{\mathcal{G}}$ are equivalent in the sense of absolute continuity. Hence, $\forall \mu \in \mathcal{P}(\Omega, \mathcal{F}, \mathcal{G}, \nu)$, $\mathcal{A}=\mathcal{A}_{\mu_{\mathcal{G}}}, \overline{\mathcal{A}}=\overline{\mathcal{A}}_{\mu_{\mathcal{G}}}$, and $\mathcal{A}_{\nu_{\mathcal{G}}}^{0}=\mathcal{A}_{\mu_{\mathcal{G}}}^{0}$. Also, let $\overline{\mathcal{P}}(\Omega, \mathcal{F}, \nu \mid \mathcal{G})(\omega)$ be defined by $(5)$. Note that the regular conditional probability measures are defined by (9). For $B_{i} \in \mathcal{A}, \mathcal{P}\left(\Omega, \mathcal{F}, \nu \mid B_{i}\right)$ denotes the set $\left\{\mu\left(\cdot \mid B_{i}\right): \mu \in \mathcal{P}(\Omega, \mathcal{F}, \mathcal{G}, \nu)\right.$ and $\mu(\cdot \mid \mathcal{G})(\cdot)$ is defined by $(9)\}$. Also, let $\overline{\mathcal{P}}\left(\Omega, \mathcal{F}, \nu \mid B_{i}\right)$ denote the set $\left\{\mu\left(\cdot \mid B_{i}\right): \mu\left(\cdot \mid B_{i}\right) \in \mathcal{P}\left(\Omega, \mathcal{F}, \nu \mid B_{i}\right)\right.$ and $\mathcal{R}(\mu(\cdot \mid \mathcal{G}) \| \nu(\cdot \mid \mathcal{G}))(\omega)<\infty, \nu_{\mathcal{G}}$-a.s. $\}$. Hence $\overline{\mathcal{P}}(\Omega, \mathcal{F}, \nu \mid \mathcal{G})(\omega)=\overline{\mathcal{P}}\left(\Omega, \mathcal{F}, \nu \mid B_{i}\right) \forall \omega \in B_{i} \in \mathcal{A}$.

Proposition 15. With the above definition, the essential infimum $h(\omega)$ defined by (6) has the following representation:

$$
\begin{aligned}
h(\omega) & =\sum_{B_{i} \in \mathcal{A}} \mu\left(\cdot \mid B_{i}\right) \in \inf _{\overline{\mathcal{P}}\left(\Omega, \mathcal{F}, \nu \mid B_{i}\right)}\left\{\mathcal{R}\left(\mu\left(\cdot \mid B_{i}\right) \| \nu\left(\cdot \mid B_{i}\right)\right)\right. \\
& \left.+\mathbf{E}^{\mu}\left[g \mid B_{i}\right]\right\} I_{B_{i}}(\omega)+\sum_{B_{i} \in \overline{\mathcal{A}}} c I_{B_{i}}(\omega),
\end{aligned}
$$

where $c$ is a nonnegative constant.

Note that the duality relationship for standard relative entropy cannot be directly applied to each atom in $\mathcal{A}$. This is because of the following constraint: $\forall \mu\left(\cdot \mid B_{i}\right) \in \overline{\mathcal{P}}\left(\Omega, \mathcal{F}, \nu \mid B_{i}\right), \mu_{\mathcal{G}} \sim \nu_{\mathcal{G}}$.

Corollary 16. Suppose the sub- $\sigma$-algebra $\mathcal{G}$ is defined by (8). Under the same conditions as in Theorem 13, the variational formula (7) holds. Furthermore, for a given $\omega$ belonging to $B_{i} \in \mathcal{A}$, we have the variational formula

$$
\begin{aligned}
& -\log \mathbf{E}^{\nu}\left[e^{-g} \mid B_{i}\right]= \\
& \inf _{\mu\left(\cdot \mid B_{i}\right) \in \overline{\mathcal{P}}\left(\Omega, \mathcal{F}, \nu \mid B_{i}\right)}\left\{\mathcal{R}\left(\mu\left(\cdot \mid B_{i}\right) \| \nu\left(\cdot \mid B_{i}\right)\right)+\mathbf{E}^{\mu}\left[g \mid B_{i}\right]\right\} .
\end{aligned}
$$

\section{CONCLUSION}

In this paper, the well-known duality relationship between free energy and relative entropy has been extended to the case where the relative entropy between two probability measures is conditioned on a given $\sigma$-algebra. An application of the duality relationship for the regular conditional relative entropy has been to solve an unconstrained optimization problem arising in a finite horizon robust state estimation problem for hidden Markov models; see (Xie et al., 2004).

\section{REFERENCES}

Boel, R.K., M.R. James and I.R. Petersen (2002). Robustness and risk-sensitive filtering. IEEE Transactions on Automatic Control 47(3), 451-461.

Charalambous, C.D., F. Rezaei and S.D. Djouadi (2003). Characterization of the optimal disturbance attenuation for nonlinear stochastic uncertain systems. In: Proceedings of the 42nd IEEE Conference on Decision \& Control.

Chow, Y.S. and H. Teicher (1988). Probability Theory, independence, interchangeability, martingales. 2nd ed.. Springer Verlag.

Dai Pra, P., L. Meneghini and W.J. Runggaldier (1996). Connections between stochastic control and dynamic games. Mathematics of Control, Signals, and Systems 9(4), 303-326.

Dellacherie, C. and P.A. Meyer (1982). Probability and Potentials B. North-Holland. Amsterdam. Translated by I.P. Wilson.

Deuschel, J.-D. and D.W. Stroock (1989). Large Deviations. Academic Press. San Diego.

Dey, S. and J.B. Moore (1995). Risk-sensitive filtering and smoothing for hidden Markov models. Systems $\&$ Control Letters 25, 361366.

Dupuis, P. and E. Ellis (1997). A Week Convergence Approach to the Theory of Large Deviations. Wiley, New York.

Elliott, R.J., L. Aggoun and J.B. Moore (1994). Hidden Markov Models: Estimation and Control. Springer-Verlag. New York.

Ephraim, Y.E. and N. Merhav (2002). Hidden Markov processes. IEEE Transactions on Information Theory 48(6), 1518-1569.

Kallenberg, O. (1997). Foundations of Modern Probability. Springer-Verlag.

Mitter, S.K. and N.J. Newton (2003). A variational approach to nonlinear estimation. SIAM Journal on Control and Optimization 42(5), 1813-1833.

Petersen, I.R., M.R. James and P. Dupuis (2000). Minimax optimal control of stochastic uncertain systems with relative entropy constraints. IEEE Transactions on Automatic Control 45(3), 398-412.

Shiryayev, A.N. (1984). Probability. SpringerVerlag. Translated by R.P. Boas.

Stroock, D.W. and S.R.S. Varadhan (1979). Multidimensional Diffusion Processes. SpringVerlag. New York.

Ugrinovskii, V.A. and I.R. Petersen (1999). Finite horizon minimax optimal control of stochastic partially observed time varying uncertain systems. Mathematics of Control, Signals and Systems 12, 1-23.

Wu, L.M. (1997). An introduction to large deviations. In: Several Topics in Stochastic Analysis (J.A. Yan, S.G. Peng, S.Z. Fang and L.M. Wu, Eds.). Press of Sciences of China. pp. 225-336. in Chinese, Beijing.

Xie, L., V.A. Ugrinovskii and I.R. Petersen (2004). Finite horizon robust state estimation of uncertain finite-alphabet hidden Markov models with regualr conditional relative entropy constraints. Accepted by the 43rd IEEE Conference on Decision \& Control, 2004. 